\title{
Yonsei-Barun Hospital
}

\author{
Bo-young Cho ${ }^{\bowtie}$, Sang-won Lee \\ Department of Neurosurgery, Yonsei-Barun Hospital, Seoul, Korea
}

$\triangle$ Corresponding Author: Bo-young Cho, Department of Neurosurgery, Yonsei-Barun Hospital, 300, Sadang-ro, Dongjak-gu, Seoul 07013, Korea. Tel: +82-2-1544-8235, Fax: +82-2-3482-1014 E-mail: goodi06@hanmail.net

Yonsei Barun Hospital offers highly professional and differentiated medical services in treating spine, joint, and pain. The directors and the professional medical teams in each department, including neurosurgery, orthopedics, anesthetic algology, diagnostic radiology, and family medicine, and over 100 staff members work in harmony to offer patient-centered customized treatment services under the treatment philosophy of preservation-based cutting-edge medicine. We have successfully performed more than 30,000 procedures and surgeries.

Treatment of spinal and articular diseases by Yonsei Barun Hospital shows highly satisfactory results of adopting a optimized cooperative treatment system, nonsurgical-centered treatment system, minimally invasive surgery, and other such advanced techniques.

The most significant characteristic of our specialists' cooperative system is that neurosurgery, orthopedics, anesthetic algology, and diagnostic radiology departments offer patients standardized treatment services through regular conferences and meetings for the purpose of designing more precise treatment after thorough medical examinations. Breaking from the old custom where a doctor from one department performs independent treatment, specialists from four departments now share patient cases by having meetings before beginning medical treatment every day so as to determine the most suitable and effective treatment for patients. Moreover, they offer subsidiary nourishment and immune function management programs through professional rehabilitation and medical training as well as functional medicine in order for patients to be able to perform daily activities. Post-surgery, our specialists design an individual spinal health maintenance program to maintain healthy spine conditions and minimize pain throughout patients' lives.

We are also actively conducting a broad range of clinical research. Yonsei Barun Hospital takes the lead in researching the latest treatment techniques that satisfy international standards and in performing scientific demonstrations by conducting joint research, verified by the IRB (Institutional Review Board), with large university hospitals, including Asan Medical Center
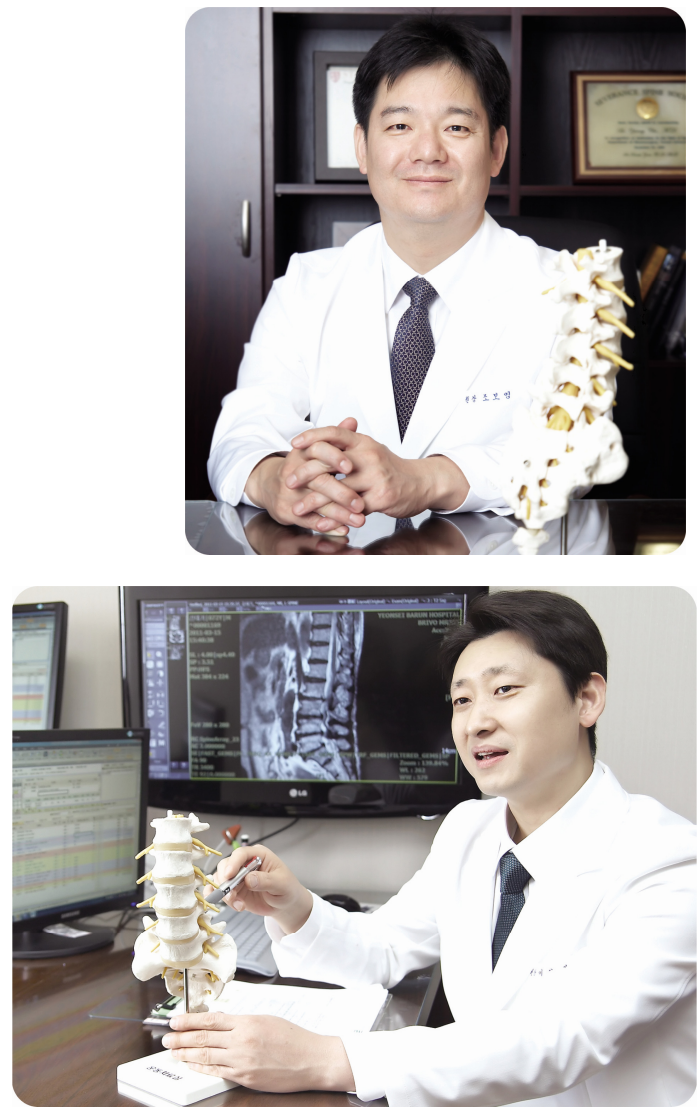

and Yonsei Medical School Severance Hospital. In these projects, Yonsei Barun Hospital takes the initiative in conducting clinical researches into balloon dilatation and the latest nonsurgical spine treatments, including intervertebral foramen endoscopic treatments. Furthermore, we continue to make presentations of the results of clinical treatment and details of researches into nonsurgical treatment at societies in and outside of Korea each year, including The Korean Neurosurgical Society, The Korean Society of Minimally Invasive Spine Surgery (KOMISS), World Congress of Minimally Invasive Pine Surgery \& Techniques (WCMISST), and The Korean Neuro-Pain Society.

Yonsei Barun Hospital's philosophy of treatment is 'A.L.L 
Barun Spirit'. This philosophy implies our medical teams' desire to offer the most effective and advanced treatment for patients through honest and advanced medical techniques. Yonsei Barun Hospital practices three core values; 'Advanced Hospital' that leads and studies advanced techniques in treating spine and joints, 'Leadership of Staff' indicating powerful leadership towards responsibility, teamwork, and duties for patients, and 'Local Contribution'.

The founders of hospital, Dr. Cho Bo-young and Dr. Lee Sang-won are noted experts in neurosurgical spine treatment in Korea. The director Cho Bo-young graduated from Yonsei University College of Medicine and completed a training course and a fellowship course at Gangnam Severance Hospital and has built expertise while serving as the head of the neurosurgery department at The National Health Insurance Corporation Ilsan Hospital, an exchange professor at the spinal surgery department at Stanford University, and the director of Gangseo Nanoori Hospital. He is currently a clinical professor at the neurosurgery department at Yonsei University College of Medicine and a regular member of The Korean Neurosurgical Society, The Korean Spinal Neurosurgery Society, The Korean Society Of Geriatric Neurosurgery and a director of planning at The Korean Neurosurgical Society, a director at The Neurosurgical Clinic Council, and a director at The Korean Neuro-Pain Society. Lee Sang-won also completed a training course at Gangnam Severance Hospital and a fellowship course at Shinchon Severance Hospital and has paved the way for minimally invasive spinal treatment by accumulating experience and skills at Daejeon Wooridul Hospital and Nanoori Hospital. He is currently active as an academic member at The Korean Neurosurgical Society, an insurance advisor at The Korean Spinal Neurosurgery Society, an executive director at The Korean Society of Minimally Invasive Spine Surgery (KOMISS), a director at The Korean Neuro-Pain Society, and a finance director at The Korean Asso- ciation of Neurological Surgeons. These two directors of the hospital have published a book named 'Barun Spinal Revolution' which contains Yonsei Barun Hospital's knowledge regarding back treatment, the latest nonsurgical treatment and post-treat- ment management, daily preventive measures, and everything else about spinal disease.
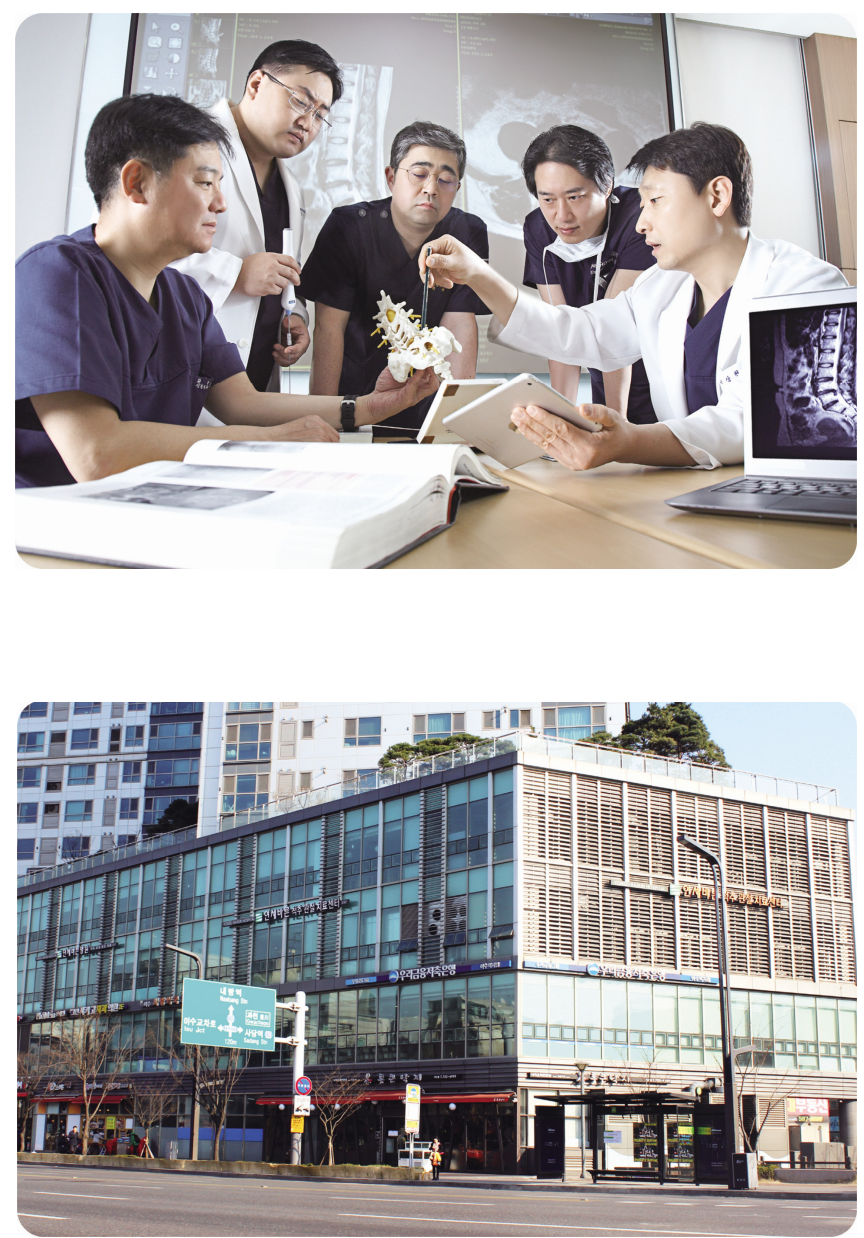